\title{
Non-invasive diagnosis of pseudoaneurysm of left ventricle
}

\author{
R. VAN MECHELEN, N. M. VAN HEMEL, AND P. P. VAN RIJK
}

From the Departments of Cardiology and Cardiovascular Surgery, St. Antonius Hospital; and the Institute of Nuclear Medicine, University Hospital, Utrecht, The Netherlands

SUMMARY A patient free of symptoms, with a pseudoaneurysm of the left ventricle after inferoposterolateral myocardial infarction, is reported. The diagnosis was established by nuclear cardiography and echocardiography of the left ventricle.

Pseudoaneurysms of the left ventricle are extremely rare. The diagnosis is usually made after death. The genesis of a pseudoaneurysm is a ventricular wall rupture, rapidly causing death by cardiac tamponade (Hurst et al., 1963; Vlodaver et al., 1975). In the rare instances of survival after myocardial rupture, pre-existing pericardial adhesions confine the bleeding to a limited space (Gueron et al., 1975). The wall of a pseudoaneurysm lacks elements of infarcted myocardium, in contrast to the wall of a true ventricular aneurysm, but is comprised of extracardiac tissue or pericardium (Van Tassel and Edwards, 1972; Gueron et al.,
1975). In this report we describe two noninvasive methods to establish the diagnosis of pseudoaneurysm of the left ventricle, which was successfully resected.

\section{Case report}

A 56-year-old man, with a history of an inferolateral myocardial infarction in November 1973 and a recurring infarction inferoposterolateral in November 1974, visited our outpatient department in September 1975 . A routine chest $x$-ray film showed a large mass, adjacent to the border of the left

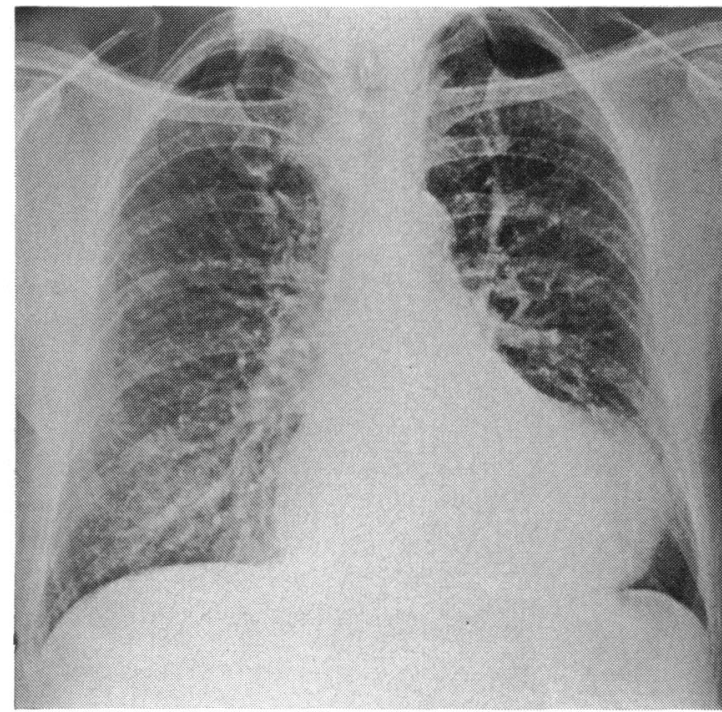

(a)

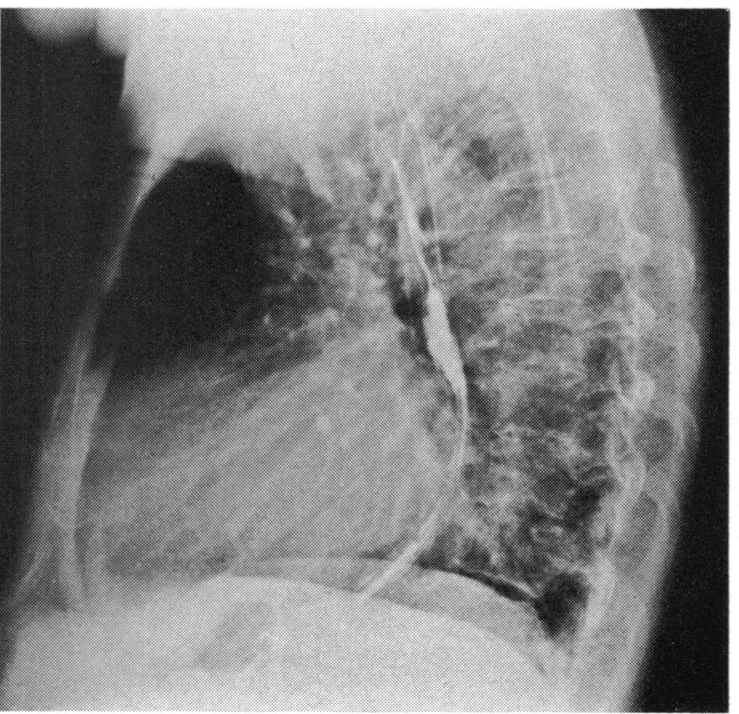

(b)

Fig. 1 Chest $\mathrm{x}$-rays showing in posteroanterior view (a) a large mass adjacent to the border of the left ventricle. The lateral projection (b) shows a dorsal displacement of the barium-filled oesophagus. 
ventricle, which had not been present on the $x$-ray film in 1974.

Ten days later the patient was admitted to our hospital for further cardiac investigation. On admission he was free of symptoms since his last infarction in November 1974. He had an administrative job, and was without cardiac complaints. Physical examination revealed a patient in good health. Blood pressure $120 / 70 \mathrm{mmHg}$, pulse 80 beats/min regular. There were neither signs of congestive heart failure, nor abnormal heart sounds or murmurs.

The chest $x$-ray film showed an enlarged heart shadow and a localised paracardial mass. Cardiothoracic ratio: 0.62 . The lateral projection demonstrated a dorsal displacement of the barium-filled oesophagus (Fig. 1a, b).

The electrocardiogram showed regular 80 beats/ min, biphasic $P$ wave in lead V1, normal conduction times; $Q$ waves were present in leads II, III, aVF, V5, V6, with a tall $R$ in V1. Flat $T$ waves were seen in leads I, II, III, aVL, and aVF. This electrocardiogram was interpreted as showing an old inferoposterolateral myocardial infarction and questionable left atrial hypertrophy.

An echocardiographic study was performed, showing an echo-free extracardiac space behind the left ventricular posterior wall (Fig. 2a). This extracardiac space could represent a pseudoaneurysm. Theoretically it also could be a pericardial cyst or extensive loculated pericardial effusion, though the latter possibilities are very unlikely.

Nuclear cardiography was performed using the high phase resolution construction technique (de Graaf and van Rijk, 1976). The patient was injected with $10 \mathrm{mCi}{ }^{99 \mathrm{~m}} \mathrm{Tc}$-albumin intravenously. After equilibrium in the circulation the spatial distribution of the radioactivity was measured with a gammacamera (Searle, LFOV), in the RAO- $15^{\circ}$ position.

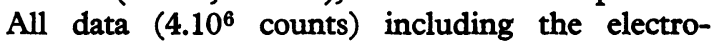
cardiogram were collected on-line by a digital acquisition system (Hewlett-Packard RTE-II) to construct a film display of the beating heart according to the high phase resolution technique (de Graaf and van Rijk, 1976). At visual examination of this film a generalised hypokinesia and extra-

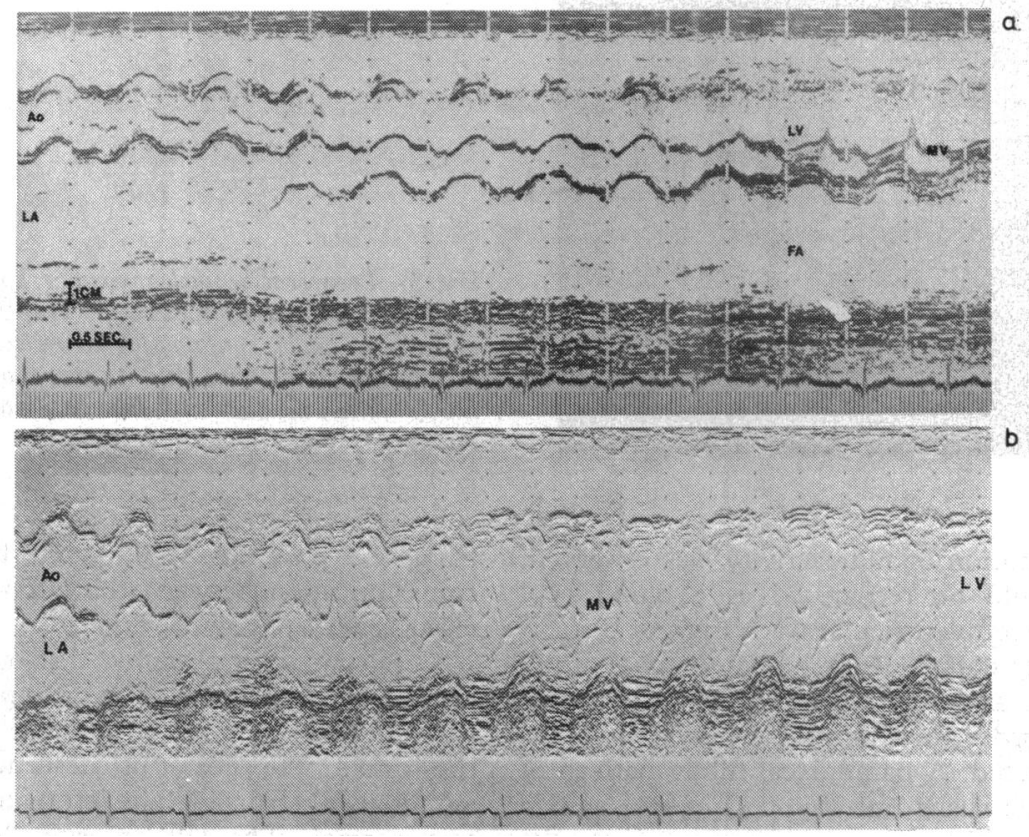

Fig. 2 (a) Preoperative study. M-mode sector from aorta (Ao) towards the apex of the left ventricle $(L V)$. There is an echo-free space posterior to the left ventricle, which is separated from it by the left ventricular posterior wall. LA, left atrium; $M V$, mitral valves; $F A$, false or pseudoaneurysm. (b) Postoperative study. The echo-free space posterior to the left ventricle is no longer present. The dimensions of the left ventricle are scarcely enlarged, with normal movements of septum and posterior wall. An intensive echo of the pericardium of the posterior wall exists, probably a postoperative effect. Ao, aorta; $M V$, mitral valves; $L V$, left ventricle; $L A$, left atrium. 


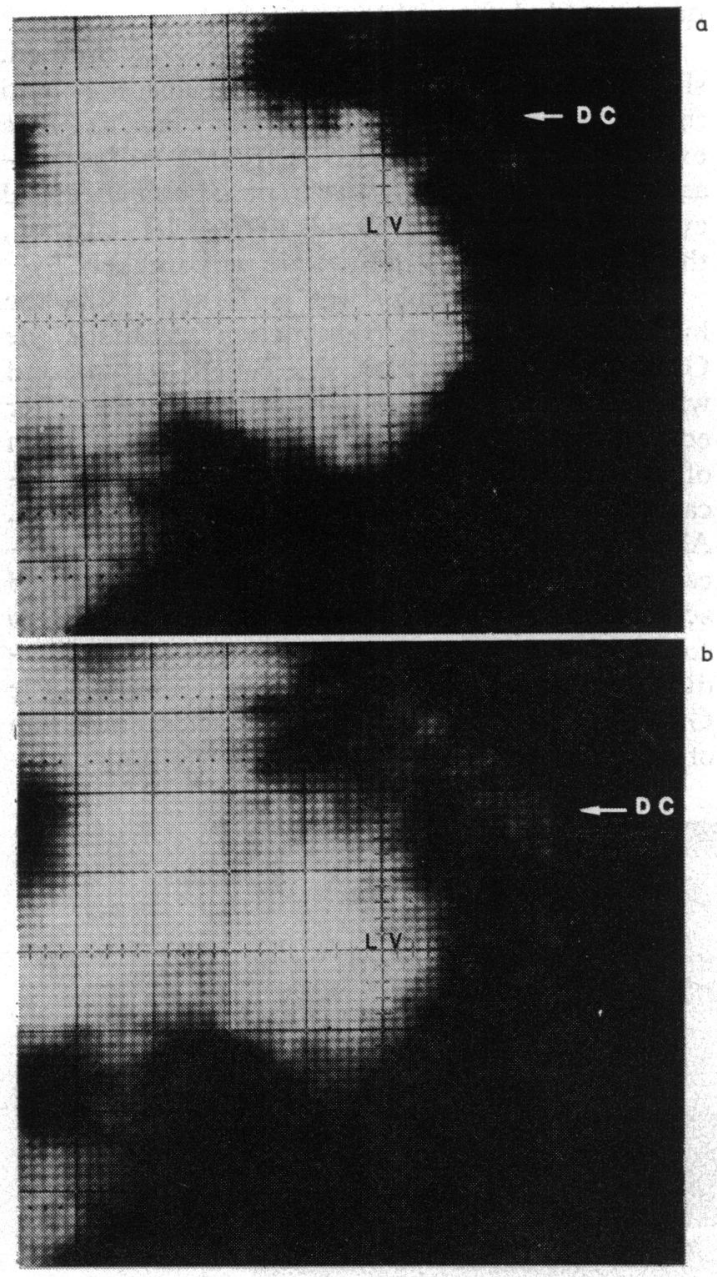

cardiac contribution of radioactivity was observed. The latter could be recognised as a blood pool adjacent to the left ventricular wall (Fig. $3 a, b$ ).

Time activity curves of the blood contents of the left ventricle and the extracardiac pool were generated in order to determine whether or not a connection existed. A paradoxical filling pattern of the extracardiac space was found (Fig. 3c), which corresponded with an open connection to the ventricle.

Cardiac catheterisation disclosed a raised left ventricular end-diastolic pressure of $24 \mathrm{mmHg}$. Cineangiography of the left ventricle was performed in right and left anterior oblique projections. In the right anterior oblique projection a severe generalised hypokinesia was observed. In the left anterior oblique projection a pseudoaneurysm, which arose

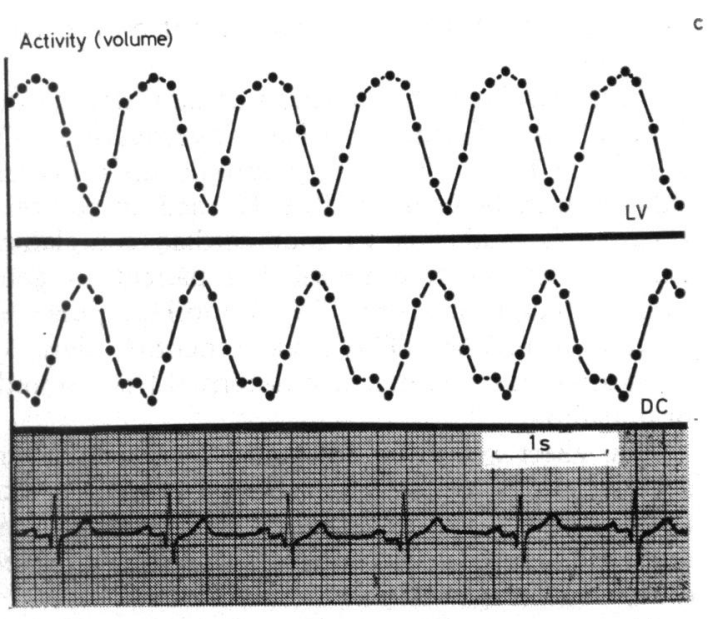

Fig. 3 Preoperative nuclear cardiographic study with ${ }^{99 \mathrm{~m}} \mathrm{Tc}$-albumin, position $\mathrm{RAO}-15^{\circ}$. (a) End-diastolic image (DC, double contour; $L V$, left ventricle). (b) Endsystolic image. (c) Time-activity curves of $L V($ top $)$ and $D C$ (middle), together with the electrocardiogram (bottom). Activity is proportional to volume.

from the posterior wall, could be visualised. $A \stackrel{\circ}{2}$ communication between the left ventricle and the $\frac{7}{0}$ extracardiac space was evident.

Coronary arteriography disclosed a dominant 0 right coronary artery, with multiple lesions of less $N$ than 35 per cent in the proximal one-third before N the crux. The posterior descending artery was subtotally obstructed. The left main coronary artery was normal. The left anterior descending artery showed 60 per cent stenosis in the proximal one- of third after the first septal and diagonal branches. The remaining vessel was normal. The circumflex artery showed 25 per cent stenosis in the proximal $\overrightarrow{\mathbb{D}}$ portion and 90 per cent stenosis in the middle $\underset{\mathbb{D}}{ }$ segment, three posterolateral branches were visual- $\varrho$ ised but not very sharply. A small first marginal branch appeared to be normal. 


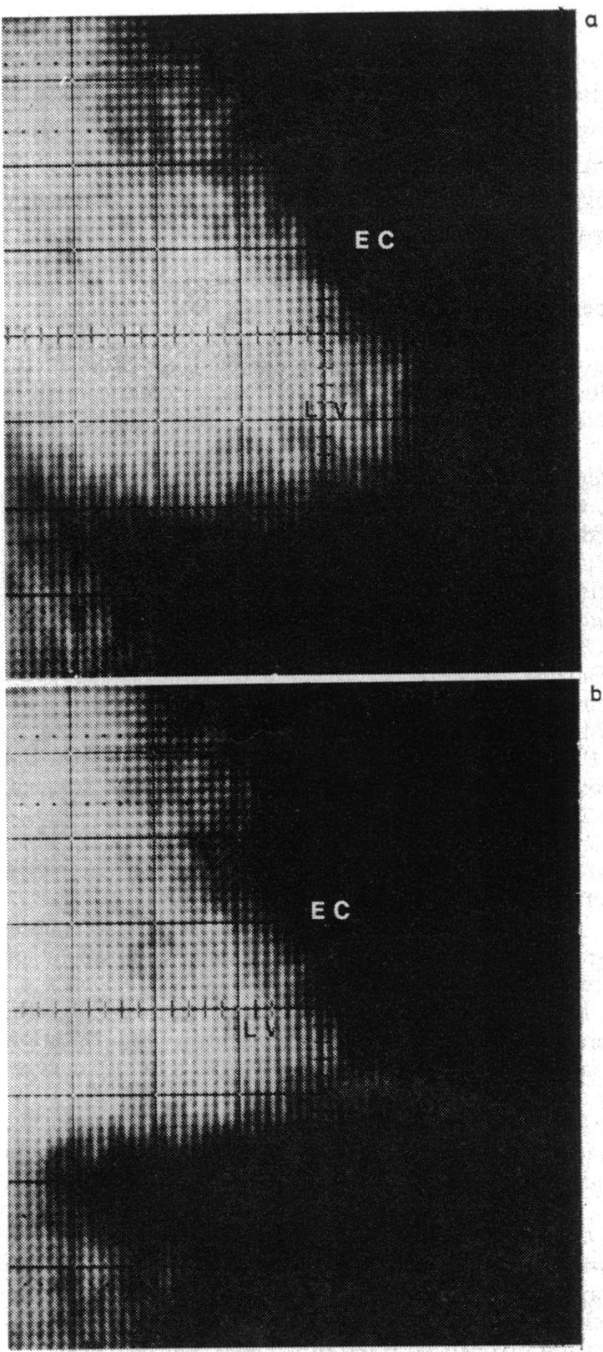

Subsequently the patient was operated upon and a large pseudoaneurysm, originating from a small perforation to the posterolateral wall, was resected. In addition a saphenous vein bypass graft was performed to the left anterior descending artery. Histology of the resected aneurysm showed that the wall consisted of non-specific fibrous material, without any identifiable myocardial element. Two weeks after the operation, the patient was discharged from the hospital. A postoperative echocardiogram (Fig. 2b) and a nuclear cardiographic study were performed in 1976.

The nuclear cardiographic study showed no extraventricular activity and a less severe hypokinesia compared with the preoperative study (Fig. 4a, b). The paradoxical filling pattern of the
Activity (volume)

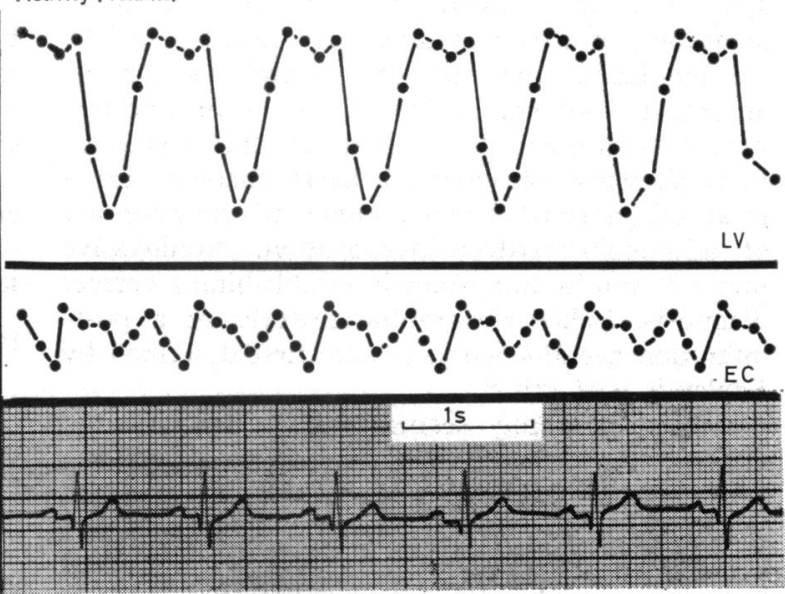

Fig. 4 Postoperative nuclear cardiographic study with ${ }^{99 \mathrm{~m}} \mathrm{Tc}$-albumin, position $R A O-15^{\circ}$. (a) End-diastolic image ( $L V$, left ventricle). (b) End-systolic image. (c) Timeactivity curves of $L V($ top $)$ and extracardiac space $(E C)$ (middle) together with the electrocardiogram (bottom). Activity is proportional to volume.

extracardiac space seen in the preoperative study could no longer be seen (Fig. 4c).

\section{Discussion}

Rupture of the myocardium after an acute myocardial infarction is occasionally compatible with survival. In such cases a pseudoaneurysm is formed (Gueron et al., 1975).

A review of the available published reports reveals 9 cases of pseudoaneurysm, in which a correct diagnosis was made in life, and successful surgical resection was performed (Smith et al., 1957; Fallah-nejad et al., 1972; MacNeil et al., 1974; Gueron et al., 1975; Harper et al., 1975; Roelandt et al., 1975; Botvinick et al., 1976). Though 
symptoms of a pseudoaneurysm are similar to symptoms of a true aneurysm of the left ventricle, we emphasise the fact that symptoms can be absent. To our knowledge this is the first patient without symptoms. In this patient, the chest $x$-ray film, showing an enlarged heart shadow and a localised paracardial mass, suggested the necessity of additional cardiac investigation. Noninvasive methods can be important in establishing a correct diagnosis. Echocardiographic features of a postinfarction pseudoaneurysm were first described by Roelandt et al. (1975).

Echocardiography seems to be a safe, though non-specific, method for the diagnosis of left ventricular pseudoaneurysm. The extracardiac space, shown as an echo-free area behind the left ventricular posterior wall, cannot be differentiated from extensive loculated pericardial effusion or a pericardial cyst (Roelandt et al., 1975).

This differentiation can be established by nuclear cardiography which is also a safe and non-invasive technique. The analogue scintigram of the heart and its surrounding tissues disclosed a blood pool adjacent to the left ventricle confirming the existence of an aneurysm, since no extraventricular activity can be shown-immediately after injection-in pericardial cyst or pericardial effusion. Furthermore, the typical halo of the latter will disappear within an hour because of mixing of radioactivity with pericardial fluid.

However, to differentiate between a pseudo aneurysm and a true aneurysm it is necessary to collect the data digitally in order to construct and analyse time-activity curves (volume curves) of the left ventricle and aneurysm. Curves obtained from a pseudo-aneurysm study will show volumes that alternate in opposite directions while in true aneurysms of the left ventricle no phase-shift will be detected.

Cineangiography of the left ventricle and selective coronary arteriography remain indispensable, primarily to determine the exact site of the ventricular wall perforation and secondly to delineate the underlying coronary artery disease.

In conclusion, the diagnosis of pseudoaneurysm of the left ventricle should be suspected on the basis of a paracardial mass in patients with a previous $c$ myocardial infarction. The diagnosis can be estab- $\vec{F}$ lished by noninvasive techniques such as echo- $\frac{\vec{\sigma}}{0}$ cardiography and nuclear cardiography.

Angiographic investigations, including coronary arteriography, remain mandatory.

\section{References}

Botvinick, E. H., Shames, D., Hutchinson, J. C., Roe, B. B. and Fitzpatrick, M. (1976). Noninvasive diagnosis of a $\vec{\omega}$ false left ventricular aneurysm with radioisotope gated $\sigma$ cardiac blood pool imaging. American fournal of Cardiology, 37, 1089-1093.

de Graaf, C. N., and van Rijk, P. P. (1976). High temporal of and high phase resolution construction techniques for $V$ cardiac motion imaging: theoretical and experimental $\infty$ comparison. In Proceedings IAEA Conference Medical $\overrightarrow{\mathrm{N}}$ Radionuclide Imaging, Los Angeles, U.S.A., IAEA-SM- 음 210/29, 377.

Fallah-nejad, M., Abelson, D. M., and Blakemore, S. (1972). Left ventricular pseudoaneurysm. Chest, 61, 90-92.

Gueron, M., Wanderman, K. L., Hirsch, M., and Borman, J. (1975). Pseudoaneurysm of the left ventricle after myocardial $\vec{\varphi}$ infarction. Fournal of Thoracic and Cardiovascular Surgery, 69, 736-742.

Harper, R. W., Sloman, G., and Westlake, G. (1975). Successful surgical resection of a chronic false aneurysm of the left ventricle. Chest, 67, 359-361.

Hurst, C. O., Fine, G., and Keyes, J. W. (1963). Pseudoaneurysm of the heart. Report of a case and review of literature. Circulation, 28, 427-436.

MacNeil, D. J., Vieweg, W. V. R., Oury, J. H., Folkerth, T. L., and Hagan, A. D. (1974). Pseudomitral regurgitation due to false aneurysm of the left ventricle treated successfully by surgery. Chest, 66, 724-726.

Roelandt, J., van dem Brand, M., Vletter, W. B., Nauta, J., and Hugenholtz, P. G. (1975). Echocardiographic diagnosis of pseudoaneurysm of the left ventricle. Circulation, 52, 466-472.

Smith, R. C., Goldberg, H., and Bailey, C. P. (1957). Pseudoaneurysm of the left ventricle: diagnosis by direct cardioangiography. Surgery, 42, 496-510.

Van Tassel, H., and Edwards, J. W. (1972). Rupture of heart complicating myocardial infarction. Chest, 61, 104-116.

Vlodaver, Z., Coe, J. I., and Edwards, J. E. (1975). True and false left ventricular aneurysms, clinicopathologic correlations. Circulation, 51, 567-572.

Requests for reprints to Dr P.P. van Rijk, University Hospital Utrecht, Institute of Nuclear Medicine, Utrecht, The Netherlands. 